\title{
Evaluation of $\boldsymbol{N}$-Butylbenzenesulfonamide (NBBS) Neurotoxicity in Sprague-Dawley Male Rats Following 27-day Oral Exposure
}

\author{
CV Rider ${ }^{1}$, KS Janardhan², D Rao², JP Morrison ${ }^{3}$, CA McPherson ${ }^{4}$, and GJ Harry 4 \\ ${ }^{1}$ Toxicology Branch, National Toxicology Program, National Institute of , NIEHS \\ 2Integrated Laboratory Systems Inc., RTP, NC \\ ${ }^{3}$ Charles River Pathology Associates, 4025 Stirrup Creek Drive, Suite 150 Durham, NC 27703 \\ ${ }^{4}$ NTP Laboratory, NIEHS, RTP, NC, 27709
}

\section{Abstract}

$N$-Butylbenzenesulfonamide (NBBS) is widely used as a plasticizer in polyacetals, polyamides, and polycarbonates and has been found in ground water and effluent from wastewater treatment sites. The compound is lipophilic and distributes rapidly to the brain but also clears rapidly and shows little evidence of accumulation. Limited studies in the literature report neurotoxicity of NBBS in rabbits and rats. Adult Sprague-Dawley male rats (Harlan) received corn oil vehicle or NBBS (100, 200, or $400 \mathrm{mg} / \mathrm{kg} / \mathrm{d}$ ) via oral gavage (5 ml/kg bwt) daily/5 days/week for 27 days. Deaths were observed in the $400 \mathrm{mg} / \mathrm{kg} / \mathrm{d}$ dose group in the first 5 days and dosing was decreased to $300 \mathrm{mg} / \mathrm{kg} / \mathrm{d}$. No alterations were observed in gait, locomotor activity, and rearing behavior. No histological lesions were observed in the testis, seminal vesicles, coagulating gland, epididymis, and prostate. In the liver, minimal centrilobular hypertrophy was evident in all rats of the high dose group. Contrary to previous reports, there was no evidence of peripheral nerve lesions or gliosis in the hippocampus or cerebellum. mRNA levels for glial fibrillary acidic acid protein, interferon gamma, CXCR-3, intracellular adhesion molecule-1, and CD11b were not altered in the hippocampus while Iba-1 levels were decreased. These data do not support previous reports of neurotoxicity for NBBS within a 4-week exposure regimen; however, neuropathological injury occurring over an extended period of exposure cannot be ruled out and given the potential for human exposure requires further examination.

\section{INTRODUCTION}

\begin{abstract}
$N$-Butylbenzenesulfonamide (NBBS; CAS registry number 3622-84-2) is produced by the bacteria Pseudomonas sp. $A B 2$ in greenhouse soil (Kim et al., 2000), is naturally present in the bark of the $P$. africanun tree (Schleich et al., 2006), and man-made for wide use as a plasticizer in polyacetals, polycarbonates, polysulfones, and polyamides. It has been found in groundwater for over 25 years (Albaiges et al., 1986; Bruchill et al., 1983, 1991; Guardiola et al., 1989; Gross et al., 2004; Hutchins and Ward, 1984; Noordsij et al., 1985; Oros et al., 2003). The potential leaching of NBBS from food-use polyamide plastics (Skjevrak et al., 2005) and nylon-11 tubing (Gilmore et al., 2004), in addition to landfill leakage (Schwarzbauer et al., 2002; Paxeus, 2000), have raised concerns regarding potential human exposure to NBBS and public health risk.
\end{abstract}

Publisher's Disclaimer: This is a PDF file of an unedited manuscript that has been accepted for publication. As a service to our customers we are providing this early version of the manuscript. The manuscript will undergo copyediting, typesetting, and review of the resulting proof before it is published in its final citable form. Please note that during the production process errors may be discovered which could affect the content, and all legal disclaimers that apply to the journal pertain. 
NBBS is lipophilic and distributes rapidly to the brain (Kumar et al., 2007). In male Sprague-Dawley male rats, distribution follows a unidirectional blood-brain barrier transfer constant of $>0.08 \mathrm{ml} / \mathrm{sec} / \mathrm{gram}$ brain wt with no evidence of compound accumulation (Kumar et al., 2007). NBBS has been considered a neurotoxic compound primarily based upon the work from Strong and colleagues. While developing a rabbit model of aluminuminduced neuropathology, Strong et al., (1990, 1991) identified intracytoplasmic neurofilamentous lesions in neurons of control rabbits dosed repeatedly with saline into the $4^{\text {th }}$ ventricle of the brain (intracisternal (ic)). This spurious effect was considered to be due to NBBS contamination from the plastic dosing vial as 18 months of storage resulted in a concentration of $83 \mu \mathrm{g} / \mathrm{ml} \mathrm{NBBS}$ in the saline dosing solution (Strong et al., 1991). When rabbits were ic injected monthly with $100 \mu \mathrm{g}$ NBBS hyperflexia was observed within a few weeks followed, within several weeks, by splaying of the hindlimbs. By 4 months, this progressed to clonus, hypertonia, focal paralysis and impaired backpedalling. By the end of the 8-month dosing period, the animals displayed forelimb hyperreflexia, focal muscle weakness, and exaggerated tonic immobility (Strong and Garruto, 1991). However, to place the severity of effect into perspective, by comparison, the average clinical score of 4 for the NBBS dosed rabbits was significantly less than the average score of 12 for a concurrent aluminum chloride dosed group (Strong and Garruto, 1991). At 8-12 months, occasional neuroaxonal spheroids and diminished microtubule-associated protein- 2 immunoreactivity with no loss of neuronal cell bodies in the nucleus motoris lateralis and inferior olivary nucleus (ION) were observed in the NBBS dosed rabbits (Strong and Garruto, 1991; Strong et al., 1991). In comparison, intraperitoneal (ip) injections of NBBS (300 mg/kg, 3x week) for 4 months resulted in clusters of increased MAP-2 immunoreactivity dendritic processes in the spinal motor neurons of the nucleus motoris lateralis. This was accompanied by similar but less severe motor deficits (Strong and Garruto, 1991).

Studies examining NBBS neurotoxicity in the rat are limited. An acute effect on normal mobility was noted in Sprague-Dawley male rats following a series of 4 ip injections of NBBS (300 mg/kg) delivered at 6-hr intervals (total $24 \mathrm{hr}$ dose of $1.2 \mathrm{~kg} / \mathrm{kg}$ ), as observed at 20 min to 2 hrs post-injection of the initial dose (Lee et al., 1995). While the previous studies utilized ic and ip dosing, one manufacturer OECD 407 oral dosing study reported minimal degenerating fibers of the sciatic nerve in 2 out of 5 Wistar rats treated with 150 $\mathrm{mg} / \mathrm{kg} / \mathrm{d}$ for 28 days (http://ofmpub.epa.gov/oppthpv/Public_Search.PublicTabs? SECTION=1\&epcount=1\&v_rs_list=24982935). Interpretation of this data is limited however, in that there was no information available regarding the nature of the lesion or details of normal degenerative indices observed in controls and no indication of clinical signs of peripheral neuropathy. In the rabbit, structural lesions were limited to the spinal cord and brain stem nuclei with no indication of changes in other brain regions (Strong and Garruto, 1991), a more diffuse effect has been reported in the rat following acute NBBS (300 mg/kg ip) dosing. As a marker of brain injury, Cho et al., (1995) examined levels of the astrocyte intermediate filament, glial fibrillary acidic protein (GFAP), and reported an increase in protein in the in the cerebrum and cerebellum/brain stem with an increased immunoreactivity in the hippocampus.

Although NBBS has more often been assessed for its neurotoxic potential, effects have been observed in the male Wistar rat reproductive organs (i.e. histological lesions in the testis and epididymis) at oral doses of $400 \mathrm{mg} / \mathrm{kg} / \mathrm{d}$ NBBS (OECD 421 study; IUCLID5 2007, http:// www.epa.gov/hpv/pubs/summaries/nbtlbnzs/c15009rr2.pdf). At $150 \mathrm{mg} / \mathrm{kg} / \mathrm{d}$ orally for 28 days, testes weights were increased and slight centrilobular hypertrophy of hepatocytes was observed in the liver of 2 males and 3 females (http://ofmpub.epa.gov/oppthpv/ Public_Search.PublicTabs?SECTION=1\&epcount=1\&v_rs_list=24982935). However, with doses of $400 \mathrm{mg}$ or higher in both studies, signs of general toxicity were evident in decreased body weight with a few animals showing abnormal gait, respiratory distress, 
tremor, piloerection, and lethargy throughout the experimental period. When quantitative structure activity relationship (QSAR) analyses were performed, MetaDrug ${ }^{\mathrm{TM}}$ models (GeneGo Inc.) predicted multiple toxic effects including: neurotoxicity, carcinogenicity, genotoxicity, and anemia; while, Leadscope Enterprise (Leadscope Inc.) QSAR models predicted that NBBS would be negative for both neurotoxicity and reproductive toxicity. Given the paucity of data indicating neurotoxicity and reproductive toxicity for NBBS in the rat and the conflicting QSAR model predictions, the current study was undertaken to examine specific aspects of NBBS toxicity following a 27-day oral dosing regimen. Based upon previous studies in the literature, adult male Sprague-Dawley rats were examined for hindlimb dysfunction, sciatic nerve histopathology, gliosis in the hippocampus and cerebellum, male reproductive organ weights and histopathology, as well as, pathological changes in the liver. Overall, at the levels examined, the findings did not offer support for previous rat studies implicating NBBS in neurotoxicity and reproductive toxicity, minimal changes were observed in the liver but only at higher dose levels than previously reported.

\section{METHODS}

\subsection{Animals}

Thirty day-old male Sprague-Dawley rats (Harlan Labs, Dublin, VA) were housed 4 per cage and allowed to acclimate to the animal facility. Food (NIH 31) and distilled deionized water were available ad libitum. At 50 days of age, rats were randomly assigned to dose groups and received a daily oral dose (5 days a week) of either corn oil vehicle $(\mathrm{n}=8)$ or NBBS (Frinton Labs, Inc; Hainesport, NJ; purity determined as greater than 99.9\%) (100 $(\mathrm{n}=8), 200(\mathrm{n}=8)$ or $400 \mathrm{mg} / \mathrm{kg} / \mathrm{d}(\mathrm{n}=11))$ in a gavage volume of $5 \mathrm{ml} / \mathrm{kg}$ body wt. The 400 $\mathrm{mg} / \mathrm{kg} / \mathrm{d}$ dose resulted in lethality 3 rats after 1 -week of dosing and the dose was lowered to $300 \mathrm{mg} / \mathrm{kg} / \mathrm{d}$ with no further incidence of morbidity or death. Animals received 5 daily doses per week over a 27-day period. All procedures were conducted according to an animal protocol approved by the NIEHS animal care and use committee.

\subsection{Neurobehavioral Observations of Hindlimb Dysfunction}

Previous work reported that the first signs of neurotoxicity in rabbits following either ic or ip injections manifested as a change in hindlimb gait evident as early as 3 weeks of dosing (Strong et al., 1991). In addition, studies in rats suggested that gait was an early indicator of NBBS toxicity (Lee et al., 1995; IUCLID5 2007, http://www.epa.gov/hpv/pubs/summaries/ nbtlbnzs/c15009rr2.pdf). Thus, rats were monitored in the home cage twice weekly, prior to dosing and at 4 hours post-dosing, for changes in general activity level and rearing behavior. Rearing in the home cage was recorded as absent or present for rearing aided and for rearing unaided by the cage wall. On dosing-day 26 (Wednesday), 3 hrs following dosing, individual animals were observationally evaluated for changes in locomotor activity (\# grid crossing), and rearing (\# rears unassisted by chamber wall) within an open field area (70 cm diameter x $46 \mathrm{~cm}$ high Plexiglas cylinder) over a $10 \mathrm{~min}$ period. Previous studies on NBBS demonstrated that, the hindlimb gait effects due to a peripheral neuropathy were progressive, thus, $24 \mathrm{hrs}$ after dosing, gait was assessed using the following rating scale: (1) normal straight line gait with no splay (2) minor splay (turn out of the toes) with hindlimbs maintained under the body, (3) splay with hindlimbs extended out from the body, (4) similar to 3 with the addition of a wobble to the gait, and (5) full splayed hindlimbs with the inability to get/keep hindquarters under the body. Within the observation chamber, the animals were scored for gait over the first $2 \mathrm{~min}$. The observer was blind to dosing conditions. 


\subsection{Tissue Collection}

On day 27, $24 \mathrm{hrs}$ following last dosing, animals were euthanized by $\mathrm{CO}_{2}$ and tissues were rapidly collected for histological evaluation. Seminal vesicles with coagulating gland, epididymis, testes, and ventral prostate were excised, weighed, and immersed in Davidson's fixative. The liver was collected and immersion fixed in $10 \%$ neutral buffered formalin. The sciatic nerve was excised, gently attached to an index card, maintaining proximal/distal orientation, and immersion fixed in either $4 \%$ paraformaldehyde $/ 0.1 \mathrm{M}$ phosphate buffer $(\mathrm{PB} ; \mathrm{pH} 7.2)$ for paraffin embedding or $3 \%$ glutaraldehyde/PB for embedding in Spurrs resin. The brain was rapidly excised from the cranium, bisected in the mid-sagittal plane, and one hemisphere was immersion fixed in $4 \%$ paraformaldehyde/PB. The hippocampus was dissected from the contralateral hemisphere and rapidly frozen on dry ice and stored at $-80^{\circ} \mathrm{C}$.

\subsection{Histological Assessments}

For paraffin embedding, fixed tissues were rinsed with PB saline within $48 \mathrm{hr}$, routinely processed for paraffin embedding, and $8 \mu \mathrm{m}$ sections were cut and mounted on Superfrost/ Plus slides (Daigger, Wheeling, IL). Sections were stained with hematoxylin and eosin (H\&E). Longitudinal $(5 \mathrm{~mm})$ and cross sections of the sciatic nerve were post-fixed with osmium tetroxide and processed into epoxy resin. One $\mu \mathrm{m}$ sections were collected, stained with toluidine blue, and mounted on glass slides for subsequent evaluation. Multiple sections were obtained to ensure a section of sufficient quality for grading. All sections were scanned (4-8 per animal) and grading was conducted on the best 1-2 sections. Grading of nerve fiber degeneration in the sciatic nerve was performed as follows: if an average of 3 or fewer foci of degeneration were present in each section of nerve, a grade of minimal nerve fiber degeneration was assigned.

Eight $\mu \mathrm{m}$ paraffin sections of the brain were collected in the sagittal plane within 1.3 and 2.3 $\mathrm{mm}$ from the midline. Rehydrated sections were subjected to heat-induced epitope retrieval (HIER) using a decloaker (Biocare Medical, Walnut Creek, CA). Astrocytes were identified by immunohistochemistry using polyclonal rabbit anti-rat glial fibrillary acidic protein antibody (GFAP; 1:200, $1 \mathrm{hr}, 24^{\circ} \mathrm{C}$, Dako, Carpinteria, CA). Microglia were identified with a rabbit polyclonal antibody to ionized calcium-binding adaptor molecule 1 (Iba-1, 1:500, $1 \mathrm{hr}, 24^{\circ} \mathrm{C}$; Wako Chemicals, Richmond, VA). The immunoreactive product was visualized using 3,3-diaminobenzidine tetrahydrochloride (DAB; Sigma-Aldrich, St. Louis, MO) as a chromogen. Slides stained for GFAP and Iba-1 were scanned at 40x magnification using an Aperio Scanscope T2 Scanner (Aperio Technologies, Inc, Vista, CA) and viewed using Aperio Imagescope v. 6.25.0.1117. The sciatic nerve sections and H\&E stained sections were examined by light microscopy.

\section{5. qRT-PCR}

To examine changes associated with a gliotic response and possible inflammatory changes in the hippocampus, total RNA was isolated by Trizol (Invitrogen; Carlsbad, CA) and 2.5 $\mu \mathrm{g}$ used for reverse transcription (SuperScript II TM Reverse Transcriptase: Invitrogen). Quantitative real time PCR (qRT-PCR) was carried out on a Perkin Elmer ABI Prism TM 7700 Sequence Detector using $3 \mu \mathrm{l}$ cDNA as a template, in a final concentration of $1 \mathrm{X}$ Power SYBR® Green Master Mix (Applied Biosystems; Foster City, CA) and optimized forward and reverse primers for GFAP (Forward: ACCGCTTTGCTAGCTACATCGAGA Reverse: TTCTCTCCAAATCCACACGCCA), the inflammatory related markers: interferon gamma (IFN $\gamma$; Forward: TGCCAAGGCACACTCATTGAAAGC Reverse:

ATGTTGTTGCTGATGGCCTGGTTG), complement component receptor 3 (CD11b or ITGAM - integrin, alpha M; Forward: ATGCCGCGAAGATCCTAGTTGTCA Reverse: AATGGCAGCGCTAAAGCCTTCTTG), Iba-1 (Forward: 
TGTGGAAGTGATGCCTGGGAGTTA Reverse:

TCAAGTTTGGACGGCAGATCCTCA), chemokine (C-X-C motif) receptor 3 (CXCR-3; Forward: ATGAACCAACCGGACAGTGCAAACAC Reverse:

TCATGATAAGCAAGGGCAGGACGA) and GAPDH (Forward: GGGAAGCTCACTGGCATGG Reverse: CTTCTTGATGTCATCATACTTGGCAG). The $50 \mu \mathrm{l}$ reaction mixture was held at $50^{\circ} \mathrm{C}$ for $2 \mathrm{~min}, 95^{\circ} \mathrm{C}$ for $10 \mathrm{~min}$, followed by 40 cycles at $95^{\circ} \mathrm{C}$ for $15 \mathrm{~s}$, and $1 \mathrm{~min}$ at $60^{\circ} \mathrm{C}$. Amplification curves from individual qRT-PCR reactions were generated (Sequence Detection System (SDS) 1.9.1 software (Applied Biosystems), threshold cycle values determined, and mean fold changes calculated from each transcript relative to the average age-matched corn oil control using the comparative $\mathrm{C}_{\mathrm{T}}$ method (Schmittgen and Livak, 2008).

\subsection{Statistical Analysis}

Terminal body weight and organ weight data displaying a homogenous distribution of variance were analyzed by one-way ANOVA. Motor activity as measured within the open field as the total number of grid crossings and number of rearings occurring over a $10 \mathrm{~min}$ session were analyzed by a one-way ANOVA. Gait was assessed by a rating scale and analyzed using a Mann Whitney U test. Subsequent independent group mean comparisons to controls were conducted by Dunnett's test. mRNA levels for each transcript were expressed as a ratio of target gene to GAPDH for each animal and analyzed by a one-way ANOVA. Subsequent independent group mean comparisons to controls were conducted by Dunnett's test. All statistical tests were conducted using Prism software and significance levels were set at $\mathrm{p}<0.05$.

\section{RESULTS}

\subsection{Clinical signs and motor activity}

Consistent with other studies, we observed deaths in the high dose group ( $400 \mathrm{mg} / \mathrm{kg} / \mathrm{d})$ and 3 rats died between days 3-5. These animals showed no clinical signs of toxicity prior to death and no physical evidence of gavage error. On day 5, the high dose level was decreased to $300 \mathrm{mg} / \mathrm{kg}$ with no further evidence of lethality. Terminal body weights were not significantly different between vehicle control and NBBS dosed rats (Table 1). Cage-side observations did not detect any dose-related difference in the behavior or general health status of the animals with no evidence of altered activity and rearing behavior. All animals displayed rearing behavior both in the center and at the wall of the home cage. Analysis of activity level as detected by locomotor activity and rearing in an open field (Fig. 1) confirmed a lack of motor deficits with no differences detected between vehicle controls and NBBS dosed rats. Within the open field arena, assessment of gait by a rating scale detected no significant difference between groups, with all animals falling within the rating score of 1.

\subsection{Male reproductive organs and liver}

Male reproductive effects were reported in an OECD 421 study including decreased testis and epididymal weights in adult males (IUCLID5 2007, http://www.epa.gov/hpv/pubs/ summaries/nbtlbnzs/c15009rr2.pdf). In the current study, there was no evidence of decreased male reproductive organ weights (Table 1). Histological evaluation showed no significant changes in the seminal vesicles, coagulating gland, epididymis, prostate, and testis (Fig. 2A, B) in the high dose (300 mg/kg/d) NBBS group, as compared to controls. In the OECD 407 study, centrilobular hypertrophy of hepatocytes was observed at $1000 \mathrm{mg} / \mathrm{kg}$ in all animals as early as 8 days of dosing and, at $150 \mathrm{mg} / \mathrm{kg}$ for 28 days, $50 \%$ of the rats showed slight centrilobular hypertrophy of hepatocytes (http://ofmpub.epa.gov/oppthpv/ Public_Search.PublicTabs?SECTION=1\&epcount=1\&v_rs_list=24982935). In the current 
study, we observed no significant changes in the liver in the 100 and $200 \mathrm{mg} / \mathrm{kg} / \mathrm{d} \mathrm{NBBS}$ dose groups as compared to controls. However, all rats of the high NBBS dose group displayed some indication of minimal centrilobular hepatocyte hypertrophy(Fig. 2C, D; Supplemental Fig. 1) as rated by two independent pathologists.

\subsection{Sciatic nerve}

As is common with examining sciatic nerve sections, rare and sporadic occurrences of nerve fiber degenerative lesions can be observed in control tissue. In 2 out of 7 control animals, paraffin sections of the sciatic nerve displayed a rare occurrence of single to multiple vacuoles forming chains in longitudinal section, filled with variable amounts of eosinophilic globular debris (Fig. 3A). In the $100 \mathrm{mg} / \mathrm{kg} / \mathrm{d}$ dose group, this same type of lesion was observed in 1 out of 7 animals, at $200 \mathrm{mg} / \mathrm{kg} / \mathrm{d}$ dose group, it was 1 out of 8 animals, and at the $300 \mathrm{mg} / \mathrm{kg} / \mathrm{d}$ dose group (Fig. 3B), it was 2 out of 7 animals. Similarly, examination of resin-embedded cross-sections stained with toluidine blue, showed that lesions were characterized by collapsed myelin sheath with no apparent axon or, rarely, a fiber being phagocytosed by a macrophage (Fig. 3C). In longitudinal resin-embedded sections, foci of linear chains of rounded myelin debris and macrophages were observed (Fig. 3D). Overall, the presence of these foci was rare (maximum of 3 foci per animal with multiple sections examined) and could be detected in approximately $50 \%$ of the animals at each dose group, as well as controls. Therefore, all indices of degenerative changes across dosed groups and control were considered minimal background changes. Thus, there was no evidence of dosedependent or treatment-related peripheral nerve degeneration following NBBS exposure.

\subsection{Astrocyte and microglia morphology}

The previous study by Cho et al., (1995) suggested a neurotoxic response in the hippocampus and the cerebellum/brain stem of rats based upon GFAP levels or immunohistochemistry with no observation of cell death. Upon examination of the H\&E staining in the hippocampus and cerebellum, we identified no cellular changes such as cell death that are often associated with an increase in GFAP and a normal cellular architecture was observed for each brain region. Morphologically, GFAP+ astrocytes displayed long, thin, fibrous processes with a uniform distribution across the hippocampus (Fig. 4A, C). This structural morphology was observed in both control and NBBS dosed rats (Fig. 4A, C). In the cerebellar medulla, GFAP+ astrocytes displayed a ramified morphology that was similar for controls and NBBS dosed rats (Fig. 4B, D). Iba1+ microglia maintained a ramified morphology with no indication of a thickening of processes or transition to a stellate or amoeboid morphology in the hippocampus (Fig. 5A, B) and the cerebellum (Fig. $5 \mathrm{C}, \mathrm{D})$. Further examination of the brain stem detected no changes in GFAP+ astrocytes or Iba-1+ microglia (data not shown).

\section{5. qRT-PCR of glia related genes in the hippocampus}

Elevations in GFAP have been associated with glial activation and often with a neuroinflammatory response. In examining multiple markers that would be indicative of each, we observed no elevations in the hippocampus for mRNA levels of related genes. Levels of mRNA for GFAP, IFN- $\gamma$ CXCR-3, and CD11b were not altered by NBBS and remained within constitutive levels seen in the control hippocampus (Fig. 6). mRNA levels for Iba-1 in the hippocampus showed a significant $(\mathrm{p}<0.05)$ NBBS treatment effect; however, rather than an indication of activation, post-hoc analysis demonstrated a significantly $(\mathrm{p}<0.05)$ lower Iba- $1 \mathrm{mRNA}$ level in the $200 \mathrm{mg} / \mathrm{kg}$ NBBS dose group as compared to vehicle controls (Fig. 6). 


\section{DISCUSSION}

NBBS has been referred to in the literature as a "neurotoxic" compound (Strong et al., 1990; Strong et al., 1991; Kumar et al., 2007). We now demonstrate that, in the Harlan SD male rat, daily oral gavage for 5 days per week for 4 weeks did not produce clinical signs of hindlimb dysfunction such as, altered gait and rearing behavior, and ambulatory motor activity was not altered. We observed no evidence of chemical-related degeneration in the sciatic nerve. Contradictory to previous reports (Cho et al., 1995), there was no evidence of increased GFAP or morphological indications of astrocyte or microglial reactivity in the hippocampus or cerebellum. We found no evidence of histological changes in the male reproductive organs and, of the tissues examined, findings were limited to minimal centrilobular hepatocellular hypertrophy in the liver in the high dose group.

In the initial characterization studies, NBBS injections induced hyperplasia in rabbits within 3 weeks (Strong and Garruto, 1991) and, by 3-4 months, dendritic abnormalities were observed in the nucleus motoris lateralis (Strong et al., 1990; 1991). Monthly injections of NBBS $(100 \mu \mathrm{g})$ directly into the $4^{\text {th }}$ ventricle of the brain resulted in occasional neuroaxonal spheroids and diminished microtubule-associated protein-2 immunoreactivity in the ION and nucleus motoris lateralis (Strong and Garruto, 1991). Given the likely binding of NBBS to plasma proteins, the low protein concentration of the CSF $(0.15-0.19 \mathrm{mg} / \mathrm{ml})$ relative to serum $(60 \mathrm{mg} / \mathrm{ml}$ in plasma) (Altman and Dittmer, 1971) the ic injection would likely result in a delivery of the entire injected dose to the brain, as has been discussed by Kumar et al. (2007). However, similar but less severe effects have been reported in the rabbit following ip injections (Strong et al., 1991) and sciatic nerve lesions have been reported in the rat following 28 day oral dosing (http://ofmpub.epa.gov/oppthpv/Public_Search.PublicTabs? SECTION=1\&epcount=1\&v_rs_list=24982935). Kumar et al. (2007) demonstrated that NBBS uptake into the brain was very rapid achieving a concentration greater than or equal to that of the perfusion fluid within 15-30 sec. Uptake was sufficiently high that the singlepass extraction of NBBS from saline was close to $100 \%$. While the $15 \mathrm{sec} \mathrm{K}_{\text {in }}$ values for NBBS from serum perfusion was approximately $50 \%$ that from saline, the uptake from serum was also rapid with a mean cortical extraction of $46 \%$. There was no apparent brain regional distribution specificity with brain to blood NBBS concentration ratios of 2:1 in the cortex, cerebellum, and spinal cord with a background-corrected NBBS content in the brain of approximately $1700 \mathrm{ng} / \mathrm{g}$ and in the blood of $600 \mathrm{ng} / \mathrm{ml}$ following an iv injection of $1 \mathrm{mg} /$ $\mathrm{kg}$ NBBS (Kumar et al., 2007). While the need for repeat dosing over an extended period of time was initially interpreted as representative of progressive neurodegeneration, the kinetics of NBBS, the rapid distribution, clearance, and lack of persistence in the brain (Kumar et al., 2007), suggest that the pattern of clinical signs could also be interpreted as a series of acute effects occurring upon the pulse delivery of each injection. This would be consistent with the previous pattern reported in the rabbit, in the display of clinical signs after the first dose and a greater level of severity seen after each subsequent dose with a plateau between dosing intervals (Strong et al., 1991). Acute clinical signs of hindlimb dysfunction and staggering gait have been observed in the rat within 20 min of NBBS injection (Lee et al., 1995). Systematic evaluation of locomotor activity indicates an initial decrease in activity level with the first injection. With additional injections, the mean activity levels are lower than that observed in the first session; however, the high level of variability in the ambulatory and rearing measures suggests that individual animals exhibit high activity levels after the $3^{\text {rd }}$ and $4^{\text {th }}$ dose of NBBS and test session. These data are somewhat difficult to interpret in that the health of the animal deteriorates with the repeated dosing. They could represent of a lack of habituation but also may reflect a physiological activation with the clearance of NBBS. The authors speculated that the effects were due to a decrease in the area of neuronal immunoreactivity for choline acetyltransferase in the Rexed lamina IX of the lumbar spinal cord. While this could indicate a physiological change given that no neuronal death was 
reported, the data is difficult to interpret as the analysis was based upon the individual areas of labeled neurons and neuropil (controls $=45$; NBBS $=33$ ) assessed in 3 animals rather than as an average response for each animal.

Further suggestions of an early response to NBBS are derived from the study of Cho et al. (1995) indicating an elevation in GFAP protein levels in the brain and an increased hippocampal area of GFAP+ astrocytes in Wistar rats within 3-7 days of an acute ip injection of $300 \mathrm{mg} / \mathrm{kg}$ NBBS. Based upon this report, we examined the morphological characteristics of GFAP+ astrocytes and expanded the observation to include Iba-1+ microglia. We observed no evidence of an increase in GFAP+ staining or change in astrocyte morphology within the hippocampus that would be reflective of reactivity. As an astrocyte response with injury is often accompanied by a microglia response, we examined both the morphological response of microglia and associated mRNA levels for genes that would be elevated with activation and found no induction with NBBS exposure. In the initial studies in the rabbit, Strong and Garruto (1991) reported changes in dendritic processes in the ION within the brainstem after ic injections. While these subtle effects may be a unique feature of the direct brain injection, disruption in the ION could contribute to the motor dysfunction observed (Strong et al., 1991) as the synchrony of olivary discharges may contribute to coordinating and controlling motor behavior (Llinas and Sasaki, 1989; De Zeeuw et al., 1998). Climbing fibers from the inferior olive innervate the Purkinje cells of the cerebellar cortex (Oldenbeuving et al., 1999; Yeo and Hesslow, 1998). While we did not observe any motor coordination deficits, we did examine the gliotic response in the cerebellum as a potential indicator of regional activation or damage as such a response was reported in the brainstem/cerebellum by Cho et al., (1995). In the current study, we found no change in GFAP+ astrocytes or Iba-1 microglia in areas of the cerebellum occupied by ION climbing fibers that would be indicative of an injury response. We did not systematically examine the ION as brain sectioning did not allow for a uniform representation across rats. While our assessments occurred following more extended dosing that that used by Cho et al (1995), astrocyte reactivity is normally considered a prolonged response and one can make the assumption that with 27 days of dosing, any GFAP response would likely be maintained. Alternatively, the GFAP response at 3 days following an acute NBBS exposure (Cho et al., 1995) may represent a shift in the cells following cessation of dosing. In that case, future studies would need to examine the astrocyte response at a similar time point post-cessation of dosing.

From the 28-day OECD 407 manufacturer-funded study, a NOAEL was estimated at $50 \mathrm{mg}$ / $\mathrm{kg} / \mathrm{d}$ based upon a slight centrilobular hypertrophy of hepatocytes in 2 males and 3 females and reported minimal level of degeneration in sciatic nerve fibers in 2 out of 5 males at the $150 \mathrm{mg} / \mathrm{kg} / \mathrm{d}$ dose level (http://ofmpub.epa.gov/oppthpv/Public_Search.PublicTabs? SECTION=1\&epcount=1\&v_rs_list=24982935). The lack of available histopathology images from the OECD 407 study precludes the direct comparison of observed lesions however, if the minimal changes in the liver were similar, they were observed in the current study observed only in the $300 \mathrm{mg} / \mathrm{kg} / \mathrm{d}$ dose group with no evidence of such changes in either of the two lower dose groups. In contrast, examination of the sciatic nerve showed no evidence of degeneration at any of the dose levels. Examining multiple paraffin and plastic embedded sections from each animal demonstrated that morphological features of the sciatic nerve were similar across all animals and consistent with the normal predicted pattern of background lesions.

When we compared out findings with earlier reports of male reproductive system toxicity, we observed a similar pattern, oral dosing with $400 \mathrm{mg} / \mathrm{kg} / \mathrm{d}$ resulted in a general level of toxicity and lethality. In the earlier OECD 421 study, this dose level for 28 days resulted in a significant decrease in absolute testis and epididymides weights and histological changes 
(IUCLID5 2007, http://www.epa.gov/hpv/pubs/summaries/nbtlbnzs/c15009rr2.pdf).

However, this dose also produced lethargy, piloerection, hunched posture, tremor, abnormal gait, and lethality. In the current study, the dose level was decreased to $300 \mathrm{mg} / \mathrm{kg} / \mathrm{d}$ due to lethality and no male reproductive organ toxicity was observed. Neither study demonstrated effects at lower doses of 100 and $200 \mathrm{mg} / \mathrm{kg} / \mathrm{d}$. It is possible that such effects at the high dose level may be related to the natural androgen receptor (AR) antagonist properties of Pygeum africanum bark derived NBBS (Papaioannou et al., 2010). To estimate this potential, we examined receptor related outcomes for NBBS in the in vitro testing through the Tox 12 high-throughput screening program and found NBBS negative for agonist and antagonism activity in nine human nuclear receptor reporter gene cell-based assays tested in quantitative high throughput screens (qHTS); including the AR [PubChem AID 588515/588516], ER a [588514/588513], FXR [588527/588526], GR [588532/58853], PPAR $\delta$ [588534/588535], PPAR $\gamma$ [588536/588537], RXR [588544/588546], TR $\beta$ [588545/588547], and VDR [588543/588541]. Whether this reflects a dose level difference or a distinct difference between the natural product and the commercial product is not known.

Although previous reports of neurotoxicity were not supported by the findings described in the current study, the data suggest that oral delivery of NBBS displays a steep dose-response curve for general toxicity, confounding the ability to examine adverse effects in a short-term study design. With the exception of the more long-term studies in rabbits most of the very limited studies suggesting that NBBS displayed neurotoxic potential were examined at relatively short dosing periods using ip dosing (Cho et al., 1995; Lee et al., 1995) and any gait changes were often associated with more general signs of toxicity. Of interest, are the observations across rabbit and rat of an acute and transient response to NBBS dosing (Strong et al., 1991; Cho et al., 1995; Lee et al., 1995) that may indicate a neuroactive potential for NBBS. Given the potential for long-term human exposure to NBBS from wastewater (Huppert et al., 1998) and landfill seepage water (Schwarzbauer et al., 2002; Paxeus, 2000) ranging between $n g$ and $\mu \mathrm{g} / \mathrm{L}$ (Eggen et al., 2010), further work remains warranted to characterize the potential toxicity of low dose NBBS following a more prolonged oral dosing regimen and focused on targeted endpoint assessments.

\section{Supplementary Material}

Refer to Web version on PubMed Central for supplementary material.

\section{Acknowledgments}

The authors would like to acknowledge Dr. Mamta Behl, Natashia Clayton, Norris Flagler, and the Pathology Core Laboratory of NIEHS for their assistance in tissue collection, histology, and imaging analysis. This study was funded by the Division of Intramural Research and the National Toxicology Program Laboratory. Dr. Janardhan was responsible for the organ pathology review and Drs. Rao and Morrison conducted the sciatic nerve review. This article is the work product of an employee or group of employees of the National Institute of Environmental Health Sciences (NIEHS), National Institutes of Health (NIH), however, the statements, opinions or conclusions contained therein do not necessarily represent the statements, opinions or conclusions of NIEHS, NIH or the United States government.

\section{References}

Albaiges J, Casado F, Ventura F. Organic indicators of groundwater pollution by a sanitary landfill. Water Res. 1986; 20:1153-1159.

Altman, PL.; Dittmer, DS. Biology Data Book. 2nd ed. Vol. Vol. III. Federation of American Societies for Experimental Biology; Maryland: 1974.

Cho HD, Jeong Y, Kim JG, Lee BH, Hwang SJ, Lee WY, Kim JG, Cho TS, Kim JS, Moon HH. The neurotoxicological alterations induced by narcotic drugs and industrial chemicals in the rat are 
associated with quantitative changes in glial fibrillary acidic protein. Korean J Toxicol. 1995; 11:315-327.

De Zeeuw CI, Simpson JI, Hoogenraad CC, Galjart N, Koekkoek SKE, Ruigrok TJH. Microcircuitry and function of the inferior olive. Trends Neurosci. 1998; 21:391-400. [PubMed: 9735947]

Eggen T, Moeder M, Arukwe A. Municipal landfill leachates: A significant source for new and emerging pollutants. Sci Total Environ. 2010; 408:5147-5157. [PubMed: 20696466]

Gilmore TJ, Mitroshkov AV, Dresel PE, Sklarew DS. Laboratory investigation into the contribution of contaminants to ground water from equipment materials used in sampling. Ground Water Monit Remediation. 2004; 24:88-94.

Gross B, Montgomery-Brown J, Naumann A, Reinhard M. Occurrence and fate of pharmaceuticals and alkylphenol ethoxylate metabolites in an effluent dominated river and wetland. Environ Toxicol Chem. 2004; 23:2074-2083. [PubMed: 15378981]

Guardiola J, Ventura F, Rivera J. Occurrence of industrial organic pollution in a groundwater supply: Screening, monitoring and evaluation of treatment processes. Water Supply. 1989; 7:11-16.

Huppert N, Wurtele M, Hahn HH. Determination of the plasticizer N-butylbenezensulfonamide and the pharmaceutical ibuprofen in wastewater using solid phase microextraction (SPME). Fresen $\mathbf{J}$ Anal Chem. 1998; 362:529-536.

Hutchins SR, Ward CH. A predictive laboratory study of trace organic contamination of groundwater: Preliminary results. J. Hydrol. 1984; 67:223-233.

Kim KK, Kang JG, Moon S, Kang KY. Isolation and identification of antifungal Nbutylbenzenesulfonamide produced by Pseudomonas sp AB2. J Antibiot. 2000; 53:131-136. [PubMed: 10805572]

Kumar G, Smith QR, Hokari M, Parepally J, Duncan MW. Brain uptake, pharmacokinetics, and tissue distribution in the rat of neurotoxic N-butylbenzenesulfonamide. Toxicol Sci. 2007; 97:253-264. [PubMed: 17369196]

Lee WY, Hwang SJ, Cho DH, Kim JS. Behavioral changes with alterations of choline acetyltransferase immunoreactivities induced by N-butylbenzenesulfonamide. Vet Hum Toxicol. 1995; 37:537-542. [PubMed: 8588290]

Llina SR, Sasaki K. Thefunctionalorganizationoftheolivo-cerebellar system as examined by multiple Purkinje cell recording. Eur J Neurosci. 1989; 1:587-603. [PubMed: 12106117]

Noordsij A, Puyker LM, Van Der Gaag MA. The quality of drinking water prepared from bank-filtered river water in the Netherlands. Sci Total Environ. 1985; 47:273-292.

Oldenbeuving AW, Eisenman LM, De Zeeuw CI, Ruigrok TH. Inferior olivary-induced expression of Fos-like immunoreactivity in the cerebellar nuclei of wild-type and Lurcher mice. Eur J Neurosci. 1999; 11:3809-3822. [PubMed: 10583470]

Oros DR, Jarman WM, Lowe T, David N, Lowe S, Davis JA. Surveillance for previously unmonitored organic contaminants in the San Francisco. Estuary Mar Pollut Bull. 2003; 46:1102-1110.

Papaioannou M, Schleich S, Roell D, Schubert U, Tanner T, Claessens F, Matusch R, Baniahmad A. NBBS isolated from Pygeum africanum bark exhibits androgen antagonistic activity, inhibits AR nuclear translocation and prostate cancer cell growth. Invest New Drugs. 2010; 28:729-743. [PubMed: 19771394]

Paxeus N. Organic compounds in municipal landfill leachates. Water Sci. Tehcnol. 2000; 42:323-333.

Ruigrok TJH, Voogd J. Organization of projections from the inferior olive to the cerebellar nuclei in the rat. J Comp Neurol. 2000; 426:209-228. [PubMed: 10982464]

Schmittgen TD, Livak KJ. Analyzing real-time PCR data by the comparative C(T) method. Nat Protoc. 2008; 3:1101-1108. [PubMed: 18546601]

Schwarzbauer J, Heim S, Brinker S, Littke R. Occurrence and alteration of organic contaminants in seepage and leakage water from a waste deposit landfill. Water Res. 2002; 36:2275-2287. [PubMed: 12108720]

Skjevrak I, Brede C, Steffensen IL, Mikalsen A, Alexander J, Fjeldal P, Herikstad H. Non-targeted multi-component analytical surveillance of plastic food contact materials: identification of substances not included in EU positive lists and their risk assessment. Food Addit Contam. 2005; 22:1012-1022. [PubMed: 16227185] 
Strong MJ, Garruto RM. Potentiation in the neurotoxic induction of experimental chronic neurodegenerative disorders: N-butyl benzenesulfonamide and aluminum chloride. Neurotoxicology. 1991; 12:415-425. [PubMed: 1745433]

Strong MJ, Garruto RM, Wolff AV, Chou SM, Fox SD, Yanagihara R. N-Butyl benzenesulfonamide: a neurotoxic plasticizer inducing a spastic myelopathy in rabbits. Acta Neuropathol. 1991; 81:235241. [PubMed: 2058361]

Strong MJ, Garruto RM, Wolff AV, Yanagihara R, Chou SM, Fox SD. N-Butylbenzenesulphonamide, a novel neurotoxic plasticizing agent. Lancet. 1990; 336:640. [PubMed: 1975419]

Yeo CH, Hesslow G. Cerebellum and conditioned responses. Trends Neurosci. 1998; 2:322-330. 

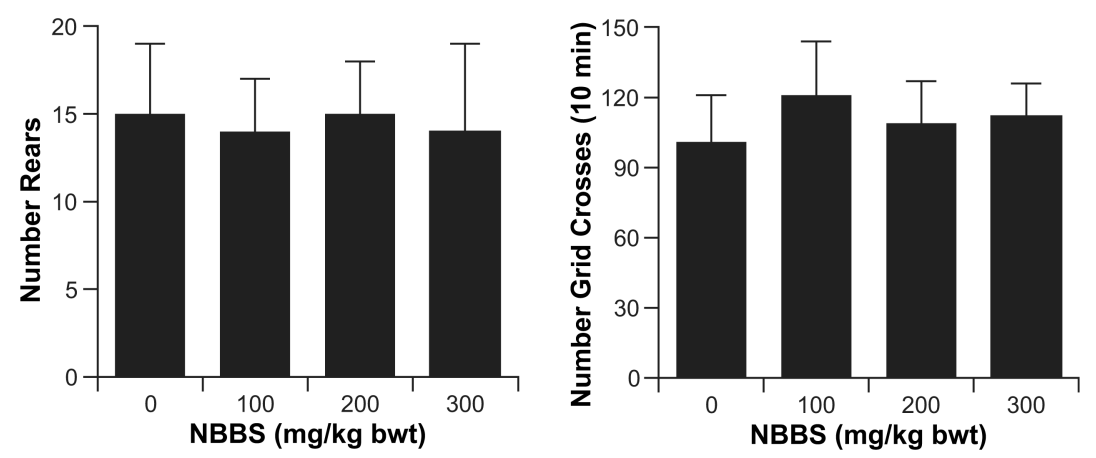

Figure 1.

Motor performance in vehicle control and NBBS dosed rats. Total number of rears occurring in an open field and total ambulatory activity in an open field as determined by number of grid crossings over a 10-min observation period. 

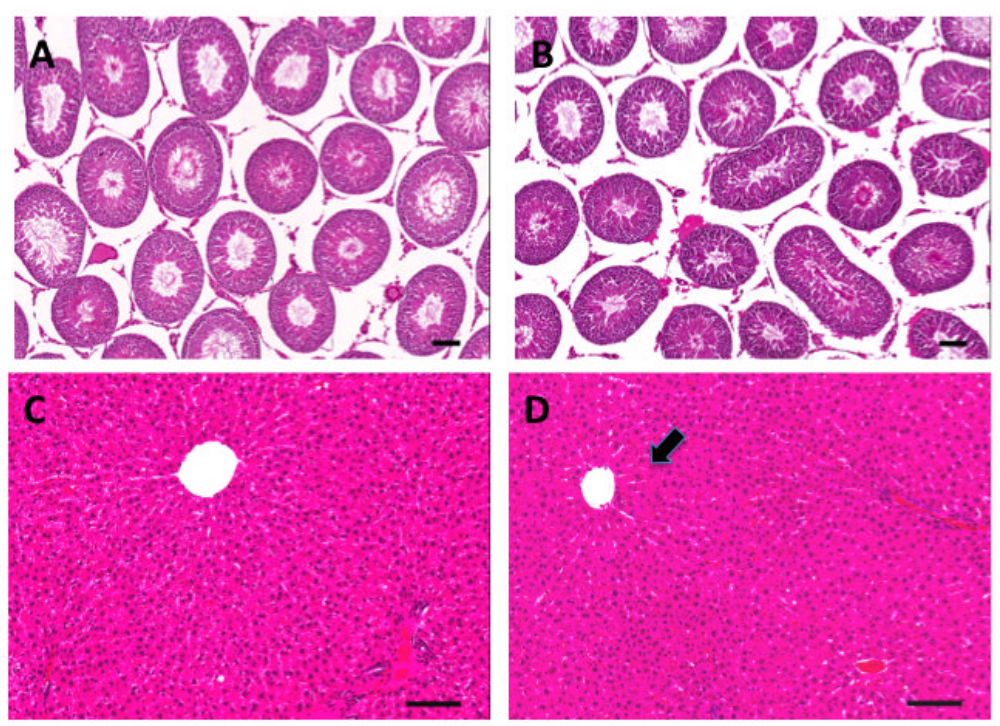

Figure 2.

Representative H\&E stained sections of the testis (A, B) and liver (C, D) of vehicle and NBBS $(300 \mathrm{mg} / \mathrm{kg} / \mathrm{d})$ at day 27 . Histologically, there are no significant changes in the testis of control (A) and treated (B) rats. Compared to (C) control liver (D) minimal centrilobular hepatocellular hypertrophy (arrow) was observed in the NBBS dosed rats. Scale bars $=25$ $\mu \mathrm{m}$ 

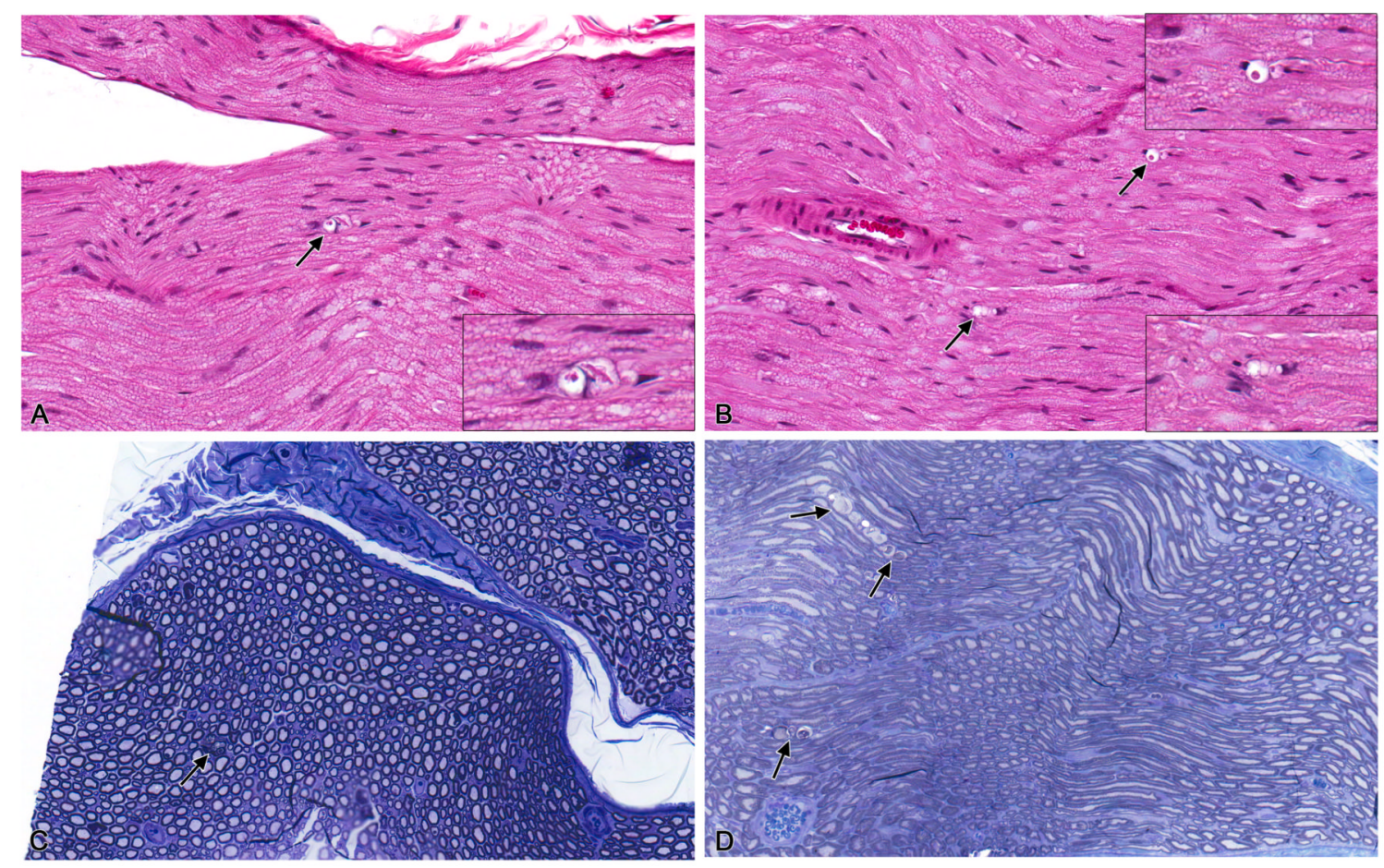

Figure 3.

Nerve fiber degeneration was observed in paraffin embedded sections of the sciatic nerve stained with $\mathrm{H} \& \mathrm{E}(\mathrm{A}, \mathrm{B})$ as well as resin embedded sections stained with toluidine blue (C, D). Arrows in A and B are highlighted in the inset figures at higher magnification. Foci of degeneration (arrows) were present equally in both controls (A, C) and NBBS (300 mg/kg/d; $\mathrm{B}, \mathrm{D})$ rats. 

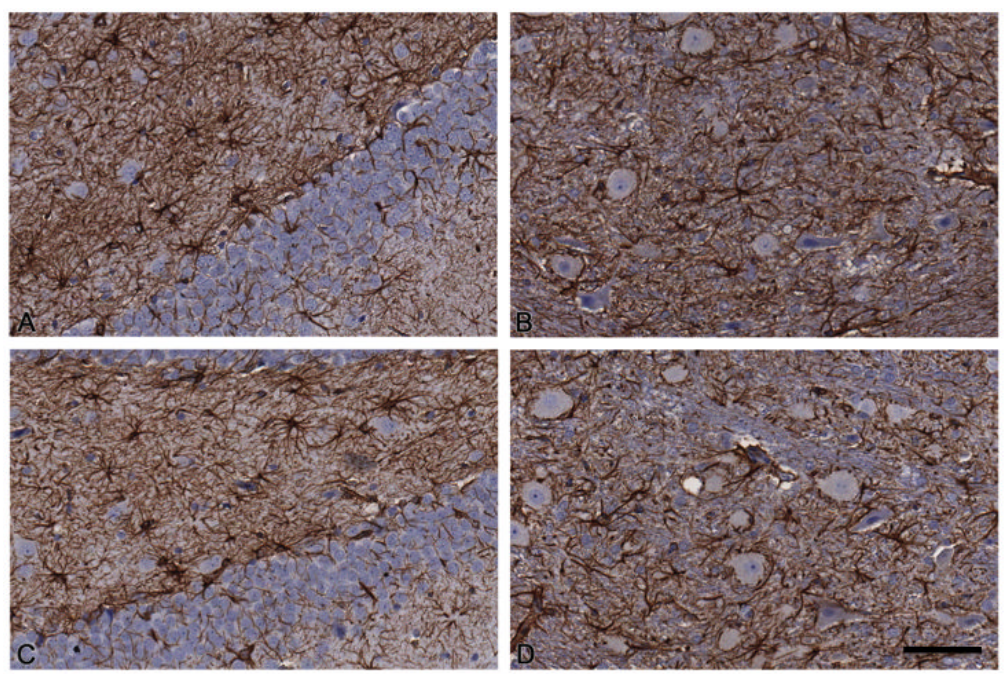

Figure 4.

Representative immunostaining for GFAP in the $(A, C)$ hippocampus and $(B, D)$ cerebellum (cerebellar nuclei region). (A) GFAP immunostaining (DAB) in the suprapyramidal blade and hilus of the dentate gyrus of (A) vehicle control and (C) NBBS $(300 \mathrm{mg} / \mathrm{kg} / \mathrm{d})$ rats at day 27 demonstrate a normal fibrous astrocyte morphology within the blade and the hilus.

Immunostaining for GFAP in the cerebellum showed similar astrocyte morphology between (B) vehicle control and (D) NBBS (300 mg/kg/d) rats. Scale bars $=50 \mu \mathrm{m}$. 

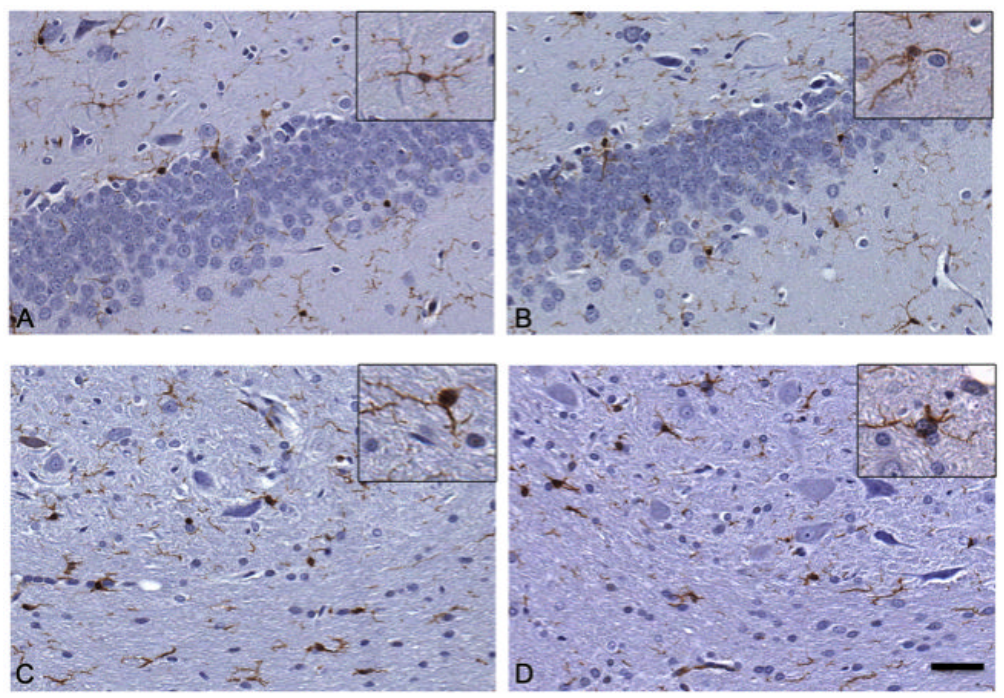

Figure 5.

Representative immunostaining for Iba-1 in the (A, B) hippocampus and (C, D) cerebellum detected with DAB and hematoxylin counterstained at day 27.

Immunostaining staining for Iba-1+ microglia within the suprapyramidal blade and hilus of the dentate gyrus of (A) vehicle control and (B) NBBS $(300 \mathrm{mg} / \mathrm{kg} / \mathrm{d})$ rats at day 27 showed similar microglia morphology with the prominence of process bearing cells. In the cerebellar nuclei region of the cerebellum, microglia displayed similar morphology between (C) vehicle controls and (D) NBBS $(300 \mathrm{mg} / \mathrm{kg} / \mathrm{d})$ rats. Scale bars $=50 \mu \mathrm{m}$. 


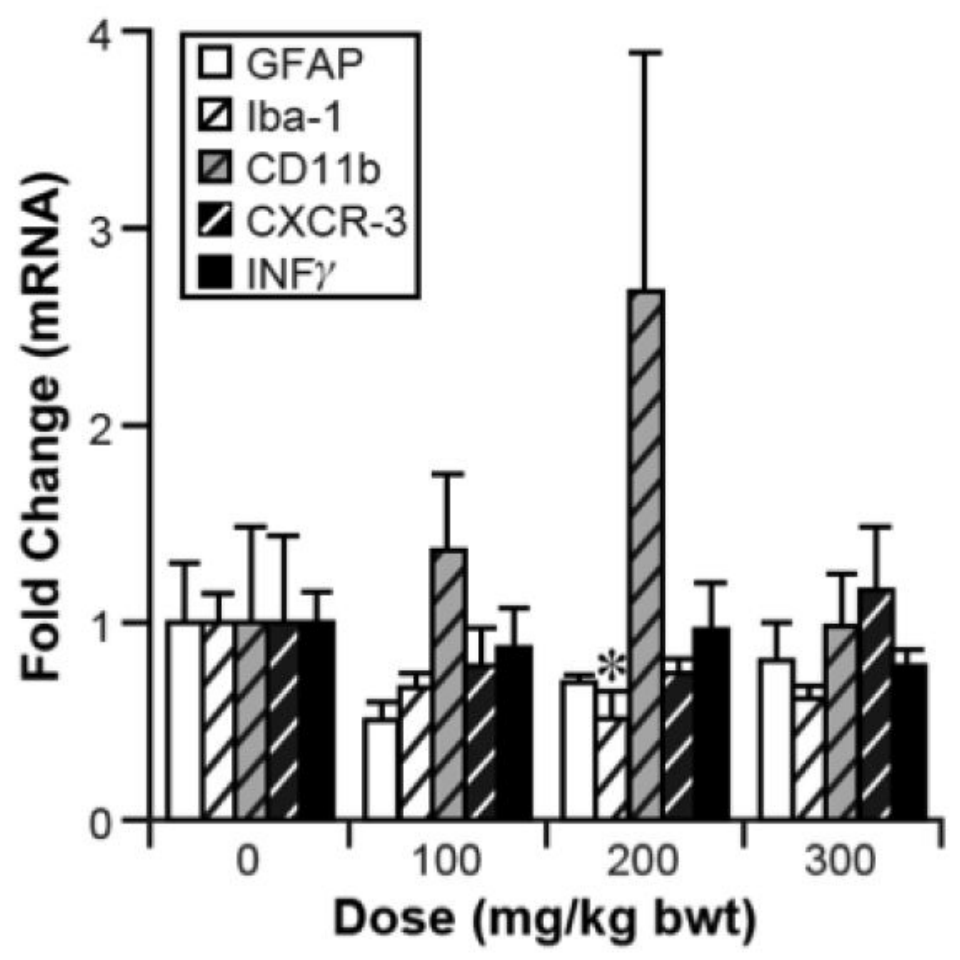

Figure 6.

Quantitative RT-PCR for GFAP, Iba-1, CD11B, CXCR-3, and INFg in the hippocampus. Sprague Dawley male rats were orally dosed with NBBS at 100,200 , or $300 \mathrm{mg} / \mathrm{kg} / \mathrm{day}$ or vehicle $(0)$ and tissue collected on day 27 . Data represents mean $+/-\operatorname{SD}(n=6) . *$ indicates significantly different $(\mathrm{p}<0.05)$ as compared to the vehicle control as determined by Dunnett's test following a significant ANOVA. 


\section{Table 1}

Body Weight and Reproductive Organ System Tissue Wet Weights

\begin{tabular}{lrrrr}
\hline Tissue Sample & Control & $\mathbf{1 0 0} \mathbf{~} \mathbf{~ g / k g}$ & $\mathbf{2 0 0} \mathbf{~ m g} / \mathbf{k g}$ & $\mathbf{4 0 0} \mathbf{~} \mathbf{g} / \mathbf{k g}$ \\
\hline Body Wt (g) & 336.5 & 353.5 & 338.8 & 318.6 \\
& $+/-24.7$ & $+/-40.8$ & $+/-14.1$ & $+/-13.7$ \\
Ventral Prostate & 0.57 & 0.54 & 0.56 & 0.51 \\
& $+/-0.08$ & $+/-0.07$ & $+/-0.08$ & $+/-0.06$ \\
Seminal Vesicles & 1.32 & 1.32 & 1.37 & 1.32 \\
& $+/-0.10$ & $+/-0.08$ & $+/-0.14$ & $+/-0.14$ \\
Right Testis & 1.83 & 1.84 & 1.87 & 1.80 \\
& $+/-0.10$ & $+/-0.09$ & $+/-0.14$ & $+/-0.08$ \\
Left Testis & 1.84 & 1.88 & 1.88 & 1.80 \\
& $+/-0.10$ & $+/-0.13$ & $+/-0.13$ & $+/-0.09$ \\
R/L Testis & 1.83 & 1.86 & 1.87 & 1.80 \\
& $+/-0.12$ & $+/-0.11$ & $+/-0.13$ & $+/-0.09$ \\
Right Epididymis & 0.48 & 0.54 & 0.53 & 0.52 \\
& $+/-0.17$ & $+/-0.02$ & $+/-0.04$ & $+/-0.02$ \\
Left Epididymis & 0.55 & 0.52 & 0.53 & 0.52 \\
R/L Epididymis & $+/-0.03$ & $+/-0.04$ & $+/-0.03$ & $+/-0.01$ \\
& 0.52 & 0.54 & 0.54 & 0.52 \\
& +-0.13 & $+/-0.03$ & $+/-0.04$ & $+/-0.02$ \\
\hline
\end{tabular}

Data represent mean gram body weight and milligram tissue wt + / - SD at necropsy. No statistically significance between dose groups was detected with ANOVA on individual weights or combined right and lest testis or right and left epididymis. 